\title{
Process optimization and evaluation of novel baicalin solid nanocrystals
}

\author{
This article was published in the following Dove Press journal: \\ International Journal of Nanomedicine \\ 8 August 2013 \\ Number of times this article has been viewed
}

\author{
Peng-Fei Yue ${ }^{1,2}$ \\ Yu Li' \\ Jing Wan' \\ Yong Wang' \\ Ming Yang' \\ Wei-Feng Zhu' \\ Chang-Hong Wang ${ }^{2}$ \\ Hai-Long Yuan ${ }^{3}$ \\ 'Key Lab of Modern Preparation of \\ TCM, Jiangxi University of Traditional \\ Chinese Medicine, Nanchang, \\ ${ }^{2}$ Institute of Chinese Materia Medica, \\ Shanghai University of Traditional \\ Chinese Medicine, Shanghai, ${ }^{3} 302$ \\ Hospital of PLA Institute of Chinese \\ Materia Medica, Beijing, People's \\ Republic of China
}

\begin{abstract}
The objective of this study was to prepare baicalin solid nanocrystals (BCN-SNS) to enhance oral bioavailability of baicalin. A Box-Behnken design approach was used for process optimization. The physicochemical properties and pharmacokinetics of the optimal BCN-SNS were investigated. Multiple linear regression analysis for process optimization revealed that the fine BCN-SNS was obtained wherein the optimal values of homogenization pressure (bar), homogenization cycles (cycles), amount of TPGS to drug (w/w), and amount of MCCS to drug (w/w) were 850 bar, 25 cycles, $10 \%$, and $10 \%$, respectively. Transmission electron microscopy and scanning electron microscopy results indicated that no significant aggregation or crystal growth could be observed in the redispersed freeze-dried BCN-SNS. Differential scanning calorimetry and X-ray diffraction results showed that $\mathrm{BCN}$ remained in a crystalline state. Dissolution velocity of the freeze-dried BCN-SNS powder was distinctly superior compared to those of the crude powder and physical mixture. The bioavailability of $\mathrm{BCN}$ in rats was increased remarkably after oral administration of BCN-SNS $(P<0.05)$, compared with those of BCN or the physical mixture. The SNS might be a good choice for oral administration of poorly soluble BCN, due to an improvement of the bioavailability and dissolution velocity of BCN-SNS.
\end{abstract}

Keywords: baicalin, solid nanocrystals, optimization, in vivo/vitro evaluation

\section{Introduction}

Baicalin (BCN; 7-D-gluconic acid, 5,6-dihydroxy flavone) (Figure 1), one of the major bioactive flavone glucuronides present in the radix of Scutellaria baicalensis, is generally used in traditional Chinese medicine as a remedy for the treatment of inflammation, fever, and allergic diseases. ${ }^{1}$ Due to the glycosyl group on the ring, $\mathrm{BCN}$ is poorly absorbed after oral administration, which causes low bioavailability and limits its therapeutic efficacy and clinical application. ${ }^{2,3}$ Because of its poor water solubility, the oral administration of $\mathrm{BCN}$ often results in erratic pharmacological activity. The poor absorption issue of poorly water-soluble drugs is known as a severe challenge for pharmaceutical development for improving drug therapeutic effectiveness. Nanonization technology is a promising formulation strategy for poorly water-soluble drugs, which could have some appealing advantages, such as increased saturation, solubility, or drug-dissolution velocity, compared with coarse or micronized drug powder. ${ }^{46}$ So far, various nanonization formulations of $\mathrm{BCN}$ have been used to improve its bioavailability, such as nanoparticles, liposomes, and nanoemulsions. ${ }^{7-9}$ However, these approaches still had some disadvantages, such as poor entrapment efficiency and low drug-loading, and were not able to give satisfactory results for $\mathrm{BCN}$ as expected.
Correspondence: Peng-Fei Yue; Ming Yang Key Lab of Modern Preparation of TCM. Jiangxi University of Traditional Chines Medicine, RD 818 Xingwan, Nanchang, People's Republic of China

Tel +8679187118108

Fax +86 79I 87II 8658

Email p.f.yuejxtcmi@gmail.com;

yangming16@I26.com 


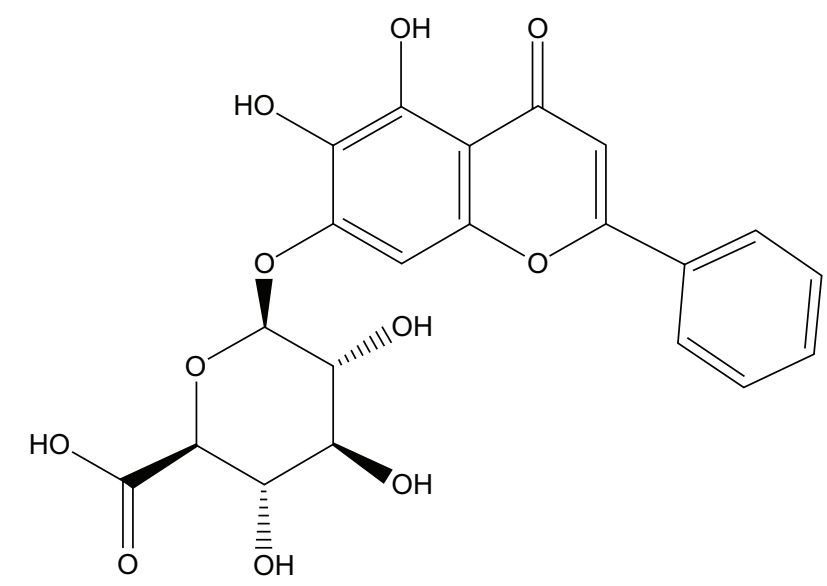

Figure I The chemical structure of baicalin.

Recently, nanosuspension (NS) technology has been successfully used to tackle the formulation problem of poorly soluble drugs. ${ }^{10,11}$ Remarkable NS properties, such as increased drug solubility and drug-loading capability (Figure 2), simple production process, easy scale-up, and universal adaptivity enable its applications in the formulation of large amounts of poorly soluble compounds. Furthermore, to improve patient convenience and physical stability issues of NS, ${ }^{12}$ it is usually preferable to transform liquid NS (LNS) into solid nanocrystals (SNS) via freeze-drying, spray-drying, or vacuum-spraying, and SNS can be redispersed into fine NS instantaneously with aqueous media in vitro or in the gastrointestinal tract. ${ }^{13}$

Commonly, NS techniques are classified as bottom-up methods ${ }^{14,15}$ or top-down methods, ${ }^{16,17}$ according to the differences in production principle. High-pressure homogenization technology is a promising method for preparing NS. However, there are many process factors that can affect the physicochemical characterizations of the obtained NS, such as particle size and particle-size distribution. Some of these factors are stabilizer or polymer amount, homogenization pressure, and homogenization circles. ${ }^{18}$ How to determine

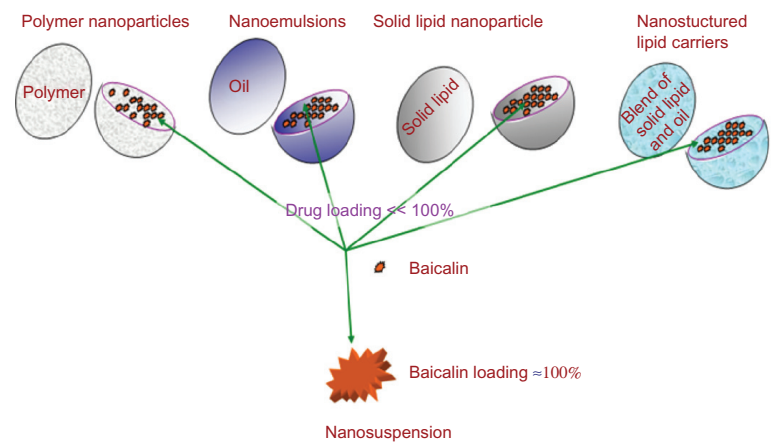

Figure 2 Comparison of nanosuspension with other nanosize delivery systems. which actual values of these variables result in a response value that is near the optimum? Box-Behnken design methodology is a well-known approach for formulation-process optimization, which can get the most information from a few well-designed experiments. ${ }^{19}$ Box-Behnken design has been comprehensively adopted to optimize the process of formulations. ${ }^{20-23}$ Those studies demonstrated the apparent advantage of Box-Behnken design utilization in the formulaoptimization process.

The main objectives of this study were: (1) to prepare acceptable BCN-NS, the Box-Behnken design approach was used for optimization of process variables, such as amounts of microcrystalline cellulose and carboxymethyl cellulose sodium (MCCS) or D- $\alpha$-tocopherol polyethylene glycol 1000 succinate (TPGS), homogenization pressure, and homogenization circles; (2) to get BCN-SNS with good redispersibility, acceptable BCN-NS was freeze-dried; furthermore, the redispersibility of BCN-SNS after freezedrying was investigated; (3) to systematically evaluate the physicochemical properties of BCN-SNS by particle-size analysis, transmission electron microscopy (TEM), differential scanning calorimetry (DSC), and powder X-ray diffraction (XRD). Furthermore, the dissolution and pharmacokinetic characteristics of BCN-SNS were also evaluated after oral administration.

\section{Materials and methods \\ Materials}

BCN was purchased from Zelang ( $>98 \%$, Nanjing, People's Republic of China). TPGS was purchased from Xi' an Healthful Biotechnology (Xi'an, People's Republic of China). MCCS (Ceolus RC-A591 NF; Asahi Kasei, Tokyo, Japan) was commercially obtained.

\section{Methods}

\section{Nanosuspension production}

BCN-NS was prepared via high-pressure homogenization. Before producing BCN-NS, BCN coarse powder $1 \%(\mathrm{w} / \mathrm{v})$, MCCS, and TPGS were dispersed in $100 \mathrm{~mL}$ water. The obtained mixture was disintegrated into coarse suspension by a high-shear homogenizer (FA25; Fluko, Shanghai, People's Republic of China) at 16,000 rpm for 5 minutes. Then, the gained coarse BCN suspension was homogenized at predetermined pressure using a piston-gap high-pressure homogenizer (AH-1000D; ATS Engineering, Seeker, Canada). The premilling step was firstly carried out at 200 bar for five cycles, and then the BCN-NSs were homogenized at 600-1000 bar for $10-30$ cycles. 


\section{Box-Behnken design}

Independent and response variables

Preliminary screening trials were carried out for evaluating the formulation process parameters of BCN-NS. Such factors as homogenization pressure, homogenization cycles, amount of TPGS to drug, and amount of MCCS to drug were identified as critical to give a product in the nanorange and with the required stability. Results from the preliminary screening trials suggested that homogenization pressure, homogenization cycles, amount of TPGS to drug, and amount of MCCS to drug were the main factors that significantly affected particle size and zeta potential of the BCN-NS.

To reduce the number of trials and attain the most information on properties of the fine NS, the screening was planned applying a Box-Behnken design. According to the principles of Box-Behnken design, homogenization pressure (A, bar), homogenization cycles (B, cycles), amount of TPGS to drug (C, \%, w/w), and amount of MCCS to drug $(\mathrm{D}, \%, \mathrm{w} / \mathrm{w})$, defined as independent values, were evaluated as four response values (Table 1), and the mean diameter $\left(\mathrm{Y}_{1}, \mu \mathrm{m}\right)$, span $\left(\mathrm{Y}_{2}\right)$, and stability index $\left(\mathrm{Y}_{3}\right)$ were defined as response values in the mathematical modeling, respectively. Each of the 29 formulations of a trial was produced three times in order to estimate the precision of the amount of TPGS to drug, amount of MCCS to drug, homogenization pressure, and homogenization cycles (see Table 2).

\section{ANOVA analysis of model}

A statistical model with interactive and polynomial terms was used to evaluate the response values using the formula ${ }^{24}$ :

Table I Process variables and their levels used in the BoxBehnken design

\begin{tabular}{llll}
\hline & \multicolumn{3}{l}{ Levels } \\
\cline { 2 - 4 } & Low & Medium & High \\
\hline Coded values & -1 & 0 & $\mathrm{I}$ \\
Independent variables & & & \\
$\mathrm{A}=$ homogenization pressure $(\mathrm{bar})$ & 600 & 800 & $\mathrm{I}, 000$ \\
$\mathrm{~B}=$ cycle numbers & 10 & 20 & 30 \\
$\mathrm{C}=$ amount of surfactant to drug $(\mathrm{w} / \mathrm{w}, \%)$ & 5 & 10 & 15 \\
$\mathrm{D}=$ amount of polymer to drug $(\mathrm{w} / \mathrm{w}, \%)$ & 5 & 10 & 15 \\
Dependent variables & & & \\
$Y_{1}=$ particle size $(\mathrm{nm})$ & & & \\
$Y_{2}=$ span & & & \\
$Y_{3}=$ stability index & & & \\
\hline
\end{tabular}

Table 2 The Box-Behnken design and the corresponding responded measurements

\begin{tabular}{|c|c|c|c|c|c|c|c|}
\hline Run & A & B & C & D & $\mathbf{Y}_{1}$ & $\mathbf{Y}_{2}$ & $\mathbf{Y}_{3}$ \\
\hline I & -1 & -1 & 0 & 0 & 1.369 & 1.738 & 0.612 \\
\hline 2 & -1 & 1 & 0 & 0 & 1.387 & 1.273 & 0.637 \\
\hline 3 & -1 & 0 & 0 & -1 & 1.417 & 1.349 & 0.511 \\
\hline 4 & -1 & 0 & 0 & 1 & 1.383 & 1.53 & 0.577 \\
\hline 5 & -1 & 0 & -1 & 0 & 1.454 & 1.225 & 0.596 \\
\hline 6 & -1 & 0 & 1 & 0 & 1.105 & 1.336 & 0.592 \\
\hline 7 & 0 & 0 & -1 & -1 & 0.714 & 1.621 & 0.637 \\
\hline 8 & 0 & 0 & I & -1 & 0.574 & 1.644 & 0.677 \\
\hline 9 & 0 & 0 & -1 & 1 & 0.603 & 1.591 & 0.728 \\
\hline 10 & 0 & 0 & I & I & 0.708 & I.672 & 0.795 \\
\hline 11 & 0 & -1 & -1 & 0 & 1.226 & 2.818 & 0.754 \\
\hline 12 & 0 & 1 & -1 & 0 & 0.655 & 1.515 & 0.734 \\
\hline 13 & 0 & -1 & I & 0 & 0.736 & 2.839 & 0.782 \\
\hline 14 & 0 & I & I & 0 & 0.566 & 1.569 & 0.792 \\
\hline 15 & 0 & -1 & 0 & -1 & 0.879 & 2.877 & 0.621 \\
\hline 16 & 0 & 1 & 0 & -1 & 0.67 & 1.681 & 0.653 \\
\hline 17 & 0 & -1 & 0 & 1 & 0.915 & 2.758 & 0.754 \\
\hline 18 & 0 & 1 & 0 & 1 & 0.625 & 1.536 & 0.715 \\
\hline 19 & 0 & 0 & 0 & 0 & 0.541 & 1.468 & 0.733 \\
\hline 20 & 0 & 0 & 0 & 0 & 0.503 & 1.58 & 0.742 \\
\hline 21 & 0 & 0 & 0 & 0 & 0.506 & 1.617 & 0.75 I \\
\hline 22 & 0 & 0 & 0 & 0 & 0.516 & 1.599 & 0.738 \\
\hline 23 & 0 & 0 & 0 & 0 & 0.588 & 1.655 & $0.74 I$ \\
\hline 24 & I & -1 & 0 & 0 & 0.535 & 3.251 & 0.642 \\
\hline 25 & I & 1 & 0 & 0 & 0.542 & 2.272 & 0.757 \\
\hline 26 & 1 & 0 & 0 & -1 & 0.513 & 2.617 & 0.615 \\
\hline 27 & 1 & 0 & 0 & 1 & 0.602 & 2.642 & 0.728 \\
\hline 28 & I & 0 & -1 & 0 & 0.588 & 2.783 & 0.686 \\
\hline 29 & I & 0 & I & 0 & 0.539 & 2.631 & 0.777 \\
\hline
\end{tabular}

$$
\begin{aligned}
Y= & b_{0}+\sum_{i} b_{i} x_{i}+\sum_{i j} b_{i j} x_{i} x_{j}++\sum_{j} b_{i} x_{i}^{2}+\sum_{i j} b_{i j} x_{i}^{2} x_{j} \\
& +\sum_{i j} b_{i j} x_{i} x_{j}^{2}+\sum_{j} b_{i} x_{i}^{3}+\varepsilon
\end{aligned}
$$

where $Y$ represented the response variables, $x$ represented the variables of the system, $i$ and $j$ were design variables, $b$ represented model coefficients that were estimated using a least square fit of the model to the experimental results obtained during the design runs, and $\varepsilon$ was the error.

To ensure a desirable model, test for significance of the regression model, test for significance on individual model coefficients, and test for lack of fit were performed. In order to summarize the tests performed, analysis of variance (ANOVA) was commonly used. The fitted model is adequate if the model is significant and the lack of fit is not significant.

\section{Optimization}

The desirability-function approach can be used to optimize the four response variables simultaneously. ${ }^{25}$ The desirability 
function was achieved with Design-Expert (Stat-Ease, Minneapolis, MN, USA). Each response variable can be assigned an importance relative to the other responses. Importance $\left(r_{i}\right)$ varies from the least important $(\cdot)$, a value of 1 , to the most important (........), a value of 5. If varying degrees of importance are assigned to the different response variables, the objective function is given as follows:

$$
D=\left(d_{1} r_{1} \times d_{2} r_{2} \times \cdots d_{n} r_{n}\right)^{1 / \sum} r_{i}=\left(\prod_{i=1}^{n} d_{i}^{r_{i}}\right)^{1 / \sum r_{i}}
$$

where $d_{i}$ is the partial desirability function of each response variable obtained from the transformation of the individual response variable of each experiment, $n$ is the number of response variables in the measure, and $r_{i}$ reflects the importance of each response variable. If all the importance variables are the same, the simultaneous objective function reduces to the normal form for desirability. Taking into account all of the requirements for each response, the process-variable conditions that maximize $\mathrm{D}$ can be determined. One can see that a high value of $\mathrm{D}$ is obtained only if all individual $d_{i}$ values are high.

\section{Verification}

According to the results of the optimization experiment, the predicted optimum values of process parameters were verified by five parallel experiments.

Particle size and span determined by laser diffractometry Laser diffractometry was performed on a Mastersizer Micro Plus (Malvern Instruments, Malvern, UK). The diffraction patterns were performed using the Mie model (particle refractive index $=1.53$, imaginary particle refractive index $=0.1$ ). From the resulting volume distributions, the D50 value $(=50 \%$ volume percentile) was calculated. All measurements were performed in triplicate.

\section{Stability index}

The stability of BCN-NS was evaluated by stability index (SI), ${ }^{26}$ as follows:

$$
\mathrm{SI}=\frac{D_{c}}{D_{0}} \times 100 \%
$$

where $D_{0}$ is the particle-size D50 value of the BCN-NS before centrifugation and $D_{c}$ is the corresponding D50 value of BCN-NS post-centrifugation at speed $1500 \mathrm{rpm}$ for 30 minutes. D50 means that $50 \%$ of the particles are below the given size. An SI value of near $100 \%$ usually means that $\mathrm{BCN}-\mathrm{NS}$ is more stable.

\section{Freeze-drying of BCN-NS}

The BCN-NS was transferred into a $100 \mathrm{~mL}$ vial and sucrose was added $(0 \%, 50 \%, 100 \%$, or $200 \%$, relative to the weight of $\mathrm{BCN})$. Then, the BCN-NSs were frozen by immersing the vials in liquid nitrogen. Freeze-drying of the BCN-NS was performed with a freeze-dryer (FD5 series; Gold SIM, Beijing, People's Republic of China) at $-20^{\circ} \mathrm{C}$ and at 0.10 mbar of pressure, and the vials were removed after 48 hours of drying. Then the BCN-SNS was obtained. Each formulation was produced in triplicate.

The stability of BCN-SNS after freeze-drying was evaluated applying the redispersibility index (RDI), as follows:

$$
\mathrm{RDI}=\frac{D}{D_{0}} \times 100 \%
$$

where $D_{0}$ is the volume-weighted mean particle size of the BCN-NS directly before freeze-drying and $D$ is the corresponding value of the redispersed $\mathrm{BCN}-\mathrm{NS}$ after freezedrying. An RDI value of near 100\% means that BCN-SNS obtained by freeze-drying can be completely redispersed to BCN-NS with original particle size after rehydration.

\section{Characterization of BCN-SNS}

The morphology of BCN-NS was observed by TEM (JEM1200EX; JEOL, Tokyo, Japan). One drop of BCN-NS was placed on a copper grid and stained with $2 \%$ phosphotungstic acid solution for 5 minutes. The grid was dried at room temperature and was evaluated with the electron microscope.

\section{Scanning electron microscopy}

Morphological evaluation of the coarse $\mathrm{BCN}$ and representative samples of BCN-SNS powder was performed and comparisons made with a scanning electron microscope (SEM; Nova NanoSEM 45; FEI, Hillsboro, OR, USA). All samples were evaluated on a brass stub using carbon double-sided tape. The samples were gold coated (thickness $\approx 15-20 \mathrm{~nm}$ ) with a sputter coater (Fisons, Ipswich, UK) using an electrical potential of $2.0 \mathrm{kV}$ at $25 \mathrm{~mA}$ for 10 minutes. An excitation voltage of $20 \mathrm{kV}$ was used in the experiments.

\section{Differential scanning calorimetry}

DSC measurements of the coarse BCN powder, stabilizers, BCN-SNS powder, and physical mixture were performed using a Diamond DSC (PerkinElmer, Waltham, MA, USA) equipped with an intercooler. Calibration for temperature and heat of fusion was performed with indium and tin as reference materials. The samples were evaluated in open aluminum pans and scanned under a nitrogen purge with a heating rate of 
$10^{\circ} \mathrm{C} /$ minute from $50^{\circ} \mathrm{C}$ to $300^{\circ} \mathrm{C}$ above the expected melting point. The measurement was performed in triplicate.

\section{Powder X-ray diffraction}

The characteristics of the stabilizers, coarse BCN powder, BCN-SNS powder, and physical mixture were analyzed by powder XRD (D8 Advance; Bruker Optik, Ettlingen, Germany) with $\mathrm{Cu}$ source of radiation. Measurements were carried out at a voltage of $40 \mathrm{kV}$ and $25 \mathrm{~mA}$. The scanned angle was performed from $3^{\circ} \leq 2 \vartheta \leq 60^{\circ}$, and the scanning rate was $2 \%$ minute. Measurements were carried out in triplicate.

\section{Solubility measurement of BCN-SNS}

Equilibrium solubility of coarse BCN, BCN-SNS, or physical mixture was performed by shake-flask method. ${ }^{27,28}$ Excess amounts of coarse BCN, BCN-SNS, and physical mixture were added in water and equilibrated in a shaker water bath (HZ-92 1K; Taicang Instrument, Taicang, People's Republic of China) at $37^{\circ} \mathrm{C}$ for 48 hours. Vials were sealed to avoid loss due to evaporation and shielded from light to prevent any degradation of BCN. As soon as equilibrium was reached, suspensions were firstly subjected to ultracentrifuge and then filtered through $0.22 \mu \mathrm{m}$ syringe filters. An aliquot of filtrate was determined by high-performance liquid chromatography (HPLC) to evaluate the dissolved amount of BCN.

\section{In vitro dissolution studies of BCN-SNS}

Dissolution characterization of BCN-SNS, coarse BCN powder, and physical mixture containing the same amount of BCN (100 mg) was evaluated. According to the China Pharmacopoeia Appendix XC paddle method, a dissolution apparatus (RC-8; Tianjin Guoming Medicine and Equipment, Tianjin, People's Republic of China) was used. Phosphatebuffered saline $(900 \mathrm{~mL}, \mathrm{pH} 7.4)$ at $37^{\circ} \mathrm{C}$ was used as a dissolution medium. The rotation speed of the paddles was set at $100 \mathrm{rpm}$. At predetermined time intervals $(5,10,20,30,45$, and 60 minutes), $2 \mathrm{~mL}$ samples were withdrawn and filtered through $0.22 \mu \mathrm{m}$ filter membrane immediately. Simultaneously, equal blank medium was compensated immediately after withdrawal. The amount of dissolved BCN in the sample solution was assayed by HPLC.

\section{Pharmacokinetic studies in vivo of BCN-SNS Blood-sample preparation}

Wister rats (body weight $280 \pm 20 \mathrm{~g}$ ) were supplied by the Experimental Animal Center of Jiangxi University of
Traditional Chinese Medicine (Nanchang, People's Republic of China). The experimental protocol was approved by the University Ethics Committee for the use of experimental animals and conformed to the Guide for the Care and Use of Laboratory Animals. All rats were divided randomly into two groups of six animals each. Rats were fasted for 12 hours prior to the study. BCN was administered to them by gavages (30 mg/kg of body weight) in the form of either BCN suspension (dispersed in 10\% [w/w] TPGS and MCCS aqueous solution) or BCN-NS. Blood samples of approximately $0.25 \mathrm{~mL}$ were collected by retroorbital puncture at predetermined intervals $0.25,0.5,0.75,1,1.5,2,3,4,6,8$, 10 , and 24 hours. Plasma was obtained from whole blood in heparinized tubes by centrifugation at 3,000 rpm for $15 \mathrm{~min}$ utes and frozen at $-20^{\circ} \mathrm{C}$ until analysis.

To a $100 \mu \mathrm{L}$ aliquot of plasma sample, $300 \mu \mathrm{L}$ methanol and $300 \mu \mathrm{L}$ acetonitrile were added and vortexed for 3 minutes in a $2.0 \mathrm{~mL}$ polypropylene tube. The mixture was centrifuged at 12,000 rpm for 10 minutes, and then the supernatant was transferred to a clean tube and evaporated to dryness under a nitrogen gas stream in a $37^{\circ} \mathrm{C}$ water bath. The dried residue was then redissolved in $100 \mu \mathrm{L}$ of mobile phase and centrifuged at 12,000 rpm for 10 minutes, and the aliquot of the supernatant was injected into the HPLC system for analysis. The mobile phase was modulated to acetonitrile and $0.2 \%(\mathrm{v} / \mathrm{v})$ aqueous phosphoric acid (47:53) at a flow rate of $1 \mathrm{~mL} /$ minute and a detection wavelength of $280 \mathrm{~nm}$.

\section{Statistical analysis}

The plasma concentration-versus-time profile was analyzed by statistical software (DAS 2.0; Boying, Beijing, People's Republic of China). A noncompartmental model was chosen to calculate the main pharmacokinetic parameters. The results were expressed as means \pm standard deviation. Differences in pharmacokinetic parameters among the three groups were tested by one-way ANOVA. Values of $P<0.05$ were considered statistically significant.

\section{Results and discussion Box-Behnken design}

Table 2 shows the experimental conditions of the BoxBehnken design along with the corresponding values observed for the three response values. Experimental data were fitted to the cubic model by ANOVA. The ANOVA results for the three responses are shown in Tables $3-5$. The results indicated that all the three models were significant at $95 \%$ confidence and the lack of fit was not significant. This 
Table 3 Analysis of variance for response surface quadratic model of $Y_{\text {, }}$

\begin{tabular}{|c|c|c|c|c|c|c|}
\hline Source & $\begin{array}{l}\text { Sum of } \\
\text { square }\end{array}$ & $d f$ & $\begin{array}{l}\text { Mean } \\
\text { square }\end{array}$ & $F$ & $P$-value & \\
\hline Model & 3.04 & 17 & 018 & 53.16 & $<0.0001$ & Significant \\
\hline A & I.4I & 1 & I.4I & 420.6 & $<0.0001$ & \\
\hline$A C$ & 0.022 & I & 0.022 & 6.68 & 0.0254 & \\
\hline$B C$ & 0.04 & I & 0.04 & 11.94 & 0.0054 & \\
\hline$A^{2}$ & 0.66 & I & 0.66 & 194.55 & $<0.0001$ & \\
\hline$C^{2}$ & 0.034 & I & 0.034 & 10 & 0.0090 & \\
\hline$D^{2}$ & 0.051 & I & 0.051 & 15.23 & 0.0025 & \\
\hline $\mathrm{B}^{2} \mathrm{C}$ & 0.037 & I & 0.037 & 10.99 & 0.069 & \\
\hline $\mathrm{BC}^{2}$ & 0.073 & I & 0.073 & 21.78 & 0.0007 & \\
\hline $\mathrm{BD}^{2}$ & 0.034 & I & 0.034 & 10.19 & 0.0086 & \\
\hline Residual & 0.037 & 11 & 0.004 & & & \\
\hline Lack of fit & 0.032 & 7 & 0.005 & 3.68 & 0.1129 & $\begin{array}{l}\text { Not } \\
\text { significant }\end{array}$ \\
\hline Pure error & 0.005 & 4 & 0.001 & & & \\
\hline Total & 3.08 & 28 & & & & \\
\hline
\end{tabular}

indicated that the fitted three models were adequate. The fitted models in terms of coded factors are demonstrated in Equations (3)-(5). Coefficient of determination $\left(R^{2}\right)$ values for particle size, span, and SI are 0.9880, 0.9972, and 0.9936, respectively. The closer $R^{2}$ is to 1 , the better the empirical models fit the actual data. Figure 3 shows surface-response graphs obtained using the fitted model.

$$
\begin{aligned}
Y 1= & 0.59-0.42 A-0.16 B-0.084 C-0.0072 A B \\
& +0.075 A C+0.30 A^{2}+0.13 B^{2} \\
& +0.055 C^{2}+0.16 A^{2} B+0.063 A C^{2} \\
Y 2= & 1.66+0.61 A-0.62 B+0.011 C-0.13 A B \\
& -0.066 A C+0.24 A^{2}+0.42 B^{2} \\
& +0.060 C^{2}+0.26 A^{2} B+0.10 A C^{2}
\end{aligned}
$$

Table 4 Analysis of variance for response surface quadratic model of $Y_{2}$

\begin{tabular}{lllllll}
\hline Source & $\begin{array}{l}\text { Sum of } \\
\text { square }\end{array}$ & $\boldsymbol{d} f$ & $\begin{array}{l}\text { Mean } \\
\text { square }\end{array}$ & $\boldsymbol{F}$ & P-value & \\
\hline Model & 10.52 & $\mathrm{I} 7$ & 0.62 & 232.93 & $<0.000 \mathrm{I}$ & Significant \\
$\mathrm{A}$ & 2.99 & $\mathrm{I}$ & 2.99 & $\mathrm{II} 25.6$ & $<0.000 \mathrm{I}$ & \\
$\mathrm{B}$ & $3.1 \mathrm{I}$ & $\mathrm{I}$ & $3.1 \mathrm{I}$ & $\mathrm{II} 1.64$ & $<0.000 \mathrm{I}$ & \\
$\mathrm{AB}$ & 0.66 & $\mathrm{I}$ & 0.066 & 24.85 & 0.0004 & \\
$\mathrm{AC}$ & 0.017 & $\mathrm{I}$ & 0.017 & $6.5 \mathrm{I}$ & 0.027 & \\
$\mathrm{~A}^{2}$ & 0.69 & $\mathrm{I}$ & 0.69 & $26 \mathrm{I} .44$ & $<0.000 \mathrm{I}$ & \\
$\mathrm{B}^{2}$ & $\mathrm{I} .47$ & $\mathrm{I}$ & $\mathrm{I} .47$ & 553.80 & & \\
$\mathrm{AC}^{2}$ & 0.028 & $\mathrm{I}$ & 0.028 & 10.39 & $0.008 \mathrm{I}$ & \\
Residual & 0.029 & $\mathrm{II}$ & 0.003 & & & \\
Lack of fit & 0.009 & 7 & $0.00 \mathrm{I}$ & 0.27 & 0.9362 & Not \\
& & & & & & significant \\
Pure error & 0.02 & 4 & 0.005 & & & \\
Total & 10.55 & 28 & & & & \\
\hline
\end{tabular}

Table 5 Analysis of variance for response surface quadratic model of $Y_{3}$

\begin{tabular}{lllllll}
\hline Source & $\begin{array}{l}\text { Sum of } \\
\text { square }\end{array}$ & df & $\begin{array}{l}\text { Mean } \\
\text { square }\end{array}$ & $\boldsymbol{F}$ & P-value & \\
\hline Model & 0.16 & 19 & 0.008 & 73.48 & $<0.0001$ & Significant \\
$\mathrm{A}$ & 0.016 & $\mathrm{I}$ & 0.016 & 144.74 & $<0.000 \mathrm{I}$ & \\
$\mathrm{C}$ & 0.005 & $\mathrm{I}$ & 0.005 & 41.46 & $<0.000 \mathrm{I}$ & \\
$\mathrm{D}$ & 0.02 & $\mathrm{I}$ & 0.02 & 181.65 & $<0.000 \mathrm{I}$ & \\
$\mathrm{AB}$ & 0.002 & $\mathrm{I}$ & 0.002 & 18.03 & 0.0022 & \\
$\mathrm{AC}$ & 0.002 & $\mathrm{I}$ & 0.002 & 20.09 & 0.0015 & \\
$\mathrm{BD}$ & 0.001 & $\mathrm{I}$ & 0.001 & 11.22 & 0.0085 & \\
$\mathrm{~A}^{2}$ & 0.048 & $\mathrm{I}$ & 0.048 & 431.29 & $<0.000 \mathrm{I}$ & \\
$\mathrm{C}^{2}$ & 0.002 & $\mathrm{I}$ & 0.002 & 14.94 & 0.0038 & \\
$\mathrm{D}^{2}$ & 0.017 & $\mathrm{I}$ & 0.017 & 151.94 & $<0.000 \mathrm{I}$ & \\
$\mathrm{A}^{2} \mathrm{~B}$ & 0.004 & $\mathrm{I}$ & 0.004 & 32.72 & 0.0003 & \\
AB & 0.002 & $\mathrm{I}$ & 0.002 & 12.27 & 0.0067 & \\
Residual & 0.001 & 9 & 0.0001 & & & \\
Lack of fit & 0.0008 & 5 & 0.0002 & 3.85 & $0.108 \mathrm{I}$ & Not \\
& & & & & & significant \\
Pure error & 0.0002 & 4 & 0.00004 & & & \\
Total & 0.16 & 28 & & & & \\
\hline
\end{tabular}

$$
\begin{aligned}
Y 3= & 0.74+0.066 A-0.0021 B+0.023 C+0.049 D \\
& +0.023 A B+0.024 A C-0.018 B D-0.086 A^{2} \\
& +0.0039 B^{2}+0.016 C^{2}-0.051 D^{2}+0.037 A^{2} B \\
& -0.029 A B^{2}
\end{aligned}
$$

The results corresponding to the three response values are discussed as follows.

\section{The effect of process parameters on the particle size of BCN-NS}

Figures $3 \mathrm{~A}-\mathrm{C}$ show the surface-response plot of the effect of process parameters on the particle size of BCN-NS. The surface-response plot of interaction homogenization pressure $\times$ cycles (Figure $3 \mathrm{~A}$ ) shows that the particle size was attained with homogenization pressure between 600 and 1000 bar. This increased as power increased from 600 to 800 bar. Homogenization cycles significantly affected particle size (Figure 3A). As can be seen from Figure 3A, the particle size decreased from $1.454 \mu \mathrm{m}$ to $0.503 \mu \mathrm{m}$ as the homogenization pressure and homogenization cycles increased. As can be seen from Figures 3B and C, the amount of TPGS affected particle size; particle size decreased with increasing TPGS amount. Figure 3B demonstrates that particle size decreased as the TPGS amount increased to $15 \%$.

\section{The effect of process parameters on the span of BCN-NS}

Figures 3D-F show the surface-response plot of the effect of process parameters on the span of BCN-NS. Figure 3D 

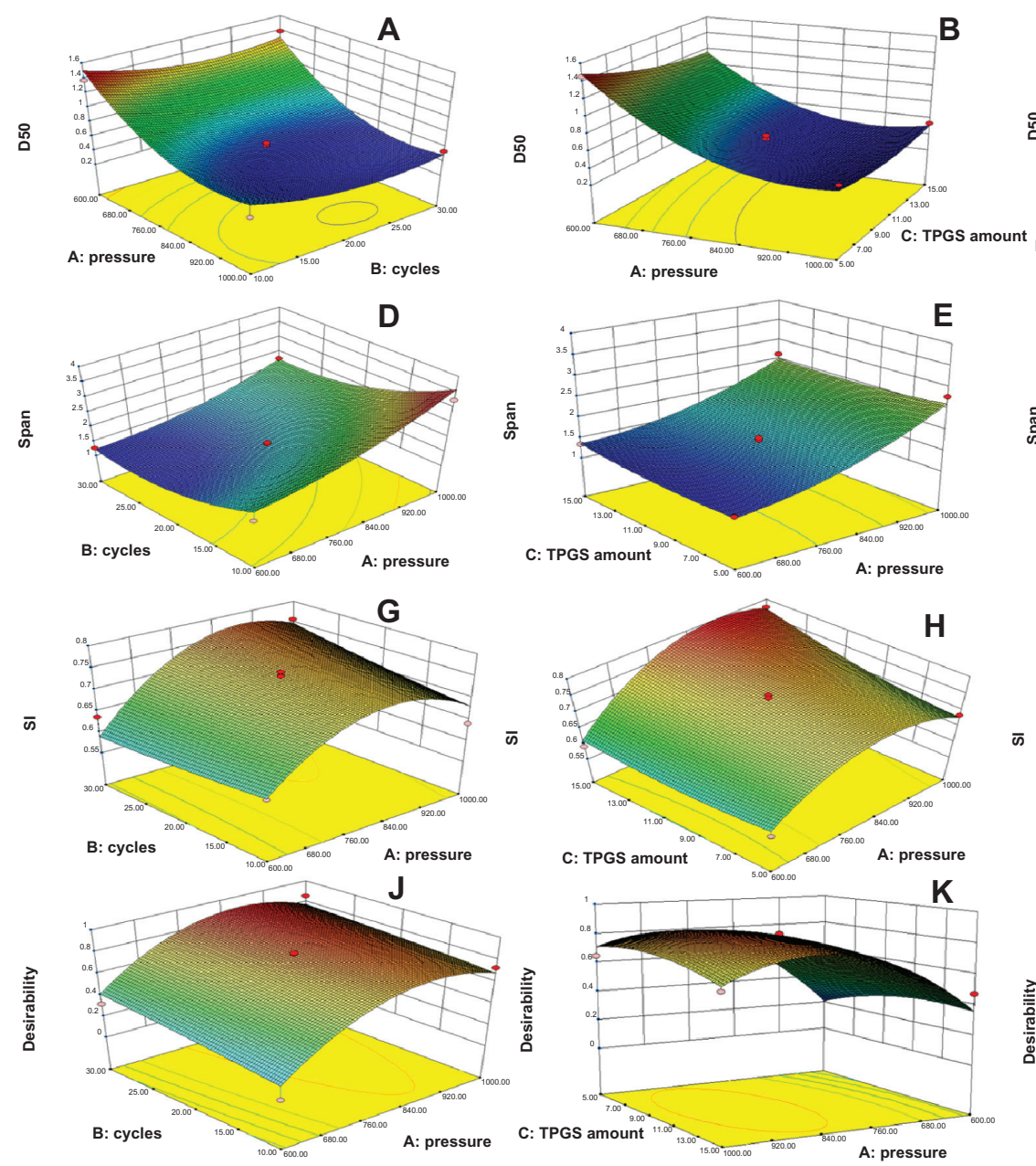

品
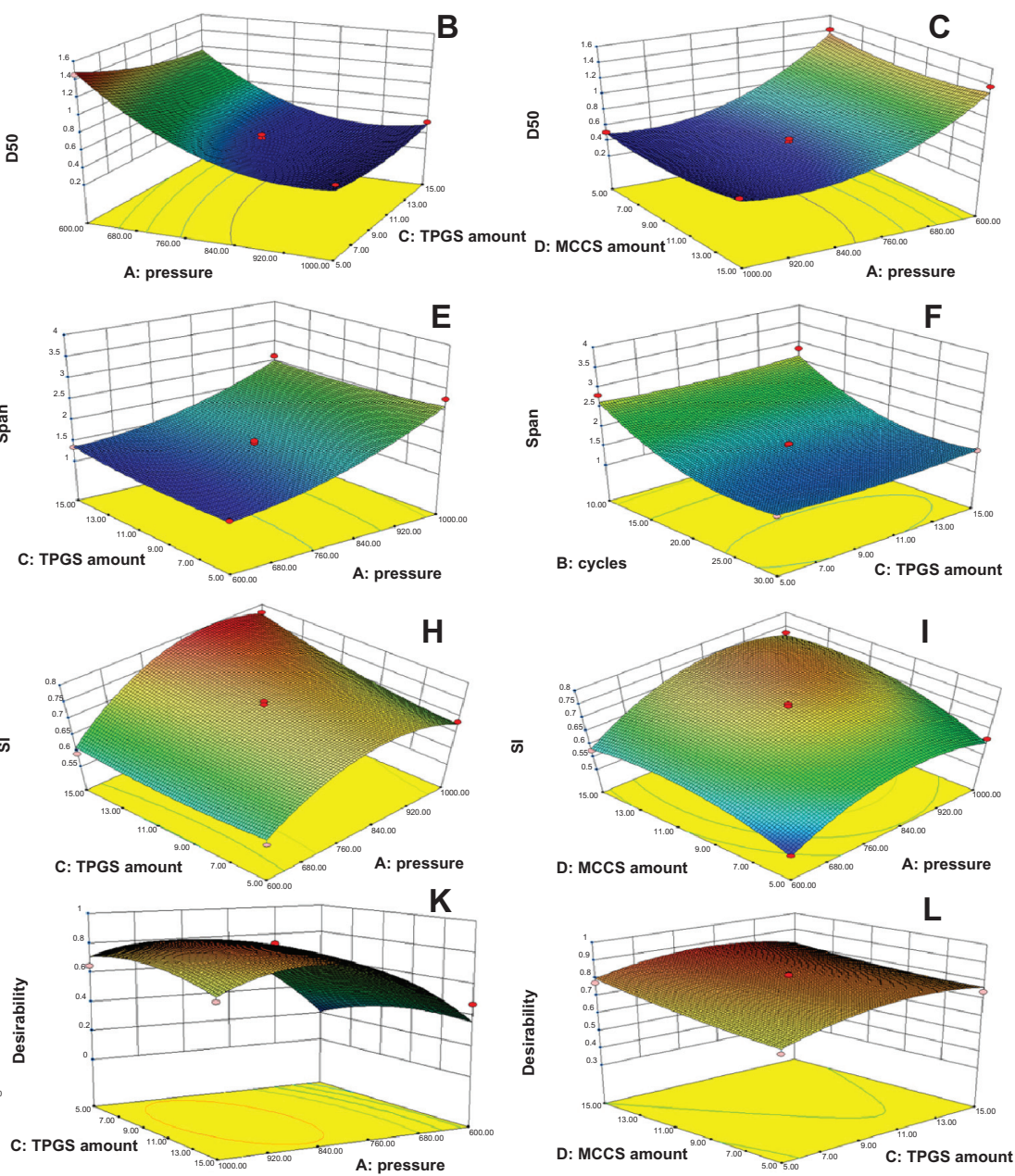

Figure 3 (A-L) Fitted surface for response values as a function of the pressure (A), homogenization cycles (B), TPGS amount (C), and MCCS amount (D). (A-C) Particle size (D50); (D-F) span; (G-I) stability index; (J-L) desirability.

Abbreviations: MCCS, microcrystalline cellulose and carboxymethyl cellulose sodium mixture; TPGS, d- $\alpha$-tocopherol polyethylene glycol I000 succinate; SI, stability index.

indicates that homogenization pressure and cycle significantly affected the span of BCN-NS. The optimum homogenization pressure was between 600 and 800 bar, while the optimum cycles were located between 20 and 25 cycles. Homogenization pressure had a significant effect on the span of BCN-NS, and the minimized span was achieved at pressure 600 bar for 20 cycles (Figure 3D). As can be seen from Figures 3E and F, the TPGS amount as well as the MCCS amount did not significantly affect the span of BCN-NS.

\section{The effect of process parameters on the stability index of BCN-NS}

Figures $3 \mathrm{G}-\mathrm{I}$ show the surface-response plot of the effect of process parameters on the SI of BCN-NS. Figure 3G shows that homogenization pressure more significantly affected the SI compared with homogenization cycles. The optimum homogenization pressure was located between 800 and 900 bar. As can be seen from Figure 3H, the SI was not significantly affected by the TPGS amount. Meanwhile, Figure 3I indicates that MCCS significantly affected the SI. The SI increased as the MCCS amount increased to $15 \%$.

\section{Desirability optimization}

The aim of optimization was to find good conditions that give the maximum SI and minimum particle size and span. The desirability-function approach was used to achieve this goal. Constraints for this optimization that were set in the software can be seen in Table 6. Figures 3J-L show the surface-response graphs of the desirability. The desirability was 0.77 . The optimum values of homogenization pressure, cycles, TPGS amount, and MCCS amount were 850 bar, 25 cycles, $10 \%$, and $10 \%$, respectively.

\section{Verification}

In order to evaluate the optimization capability of the models of the Box-Behnken design, the BCN-NSs were 
Table 6 Constraints of factors and responses for optimization

\begin{tabular}{lllll}
\hline Name & Goal & $\begin{array}{l}\text { Lower } \\
\text { limit }\end{array}$ & $\begin{array}{l}\text { Upper } \\
\text { limit }\end{array}$ & Importance \\
\hline Homogenization & Is in a range & 800 & 1000 \\
pressure (bar) & & & & \\
Homogenization cycles & Is in a range & 15 & 25 \\
TPGS amount (\%) & Is in a range & 10 & 15 & \\
MCCS amount (\%) & Is in a range & 8 & 12 & \\
Mean diameter (nm) & Minimize & & & 5 \\
Span & Minimize & & & 3 \\
Stability index & Maximize & & 4 \\
\hline
\end{tabular}

Abbreviations: MCCS, microcrystalline cellulose and carboxymethyl cellulose sodium mixture; TPGS, $d$ - $\alpha$-tocopherol polyethylene glycol 1000 succinate.

prepared according to the optimal process-variable settings, wherein $\mathrm{A}, \mathrm{B}, \mathrm{C}$, and $\mathrm{D}$ were equal to 850 bar, 20 cycles, $10 \%$, and $10 \%$, respectively. The particle size, span, and SI of BCN-NS obtained by predicted models are shown in Table 7. The results indicated that there was good agreement on preparation properties with theoretical predictions.

\section{Freeze-drying of BCN-NS}

It is well known that protectants such as polysaccharides were usually used to protect NS from the impairments of freezing and desiccation. However, apart from polysaccharides, the stabilizers were effective enough to prevent damage during freeze-drying. The results (Table 8) showed that there was no significant difference in RDI of BCN-SNS after freezedrying, compared with that of BCN-SNS with different concentrations of sucrose. BCN-SNS stabilized with TPGS and MCCS possessed good redispersibility after freezedrying. These results indicated that TPGS and MCCS were prominent stabilizers for BCN-NS, and played an important role in protecting $\mathrm{BCN}-\mathrm{NS}$ from irreversible agglomeration during freeze-drying.

\section{Morphology of BCN-SNS}

Unsuitable freeze-drying procedure of NS can induce destabilization of the particles, leading to irreversible aggregation.

Table 7 Model-predicted and observed values of particle size, span and $\mathrm{SI}$ of BCN-LNS prepared according to the optimal experimental conditions ( $A=850$ bar, $B=25, C=10 \%, D=10 \%$ ) $(n=5)$

\begin{tabular}{llll}
\hline Dependent variable & Predicted & Observed & Bias*/\% \\
\hline Mean diameter $\left(\mathrm{Y}_{1}\right)$ & $477.2 \mathrm{~nm}$ & $483.4 \mathrm{~nm}$ & -1.29 \\
Span $\left(\mathrm{Y}_{2}\right)$ & 1.307 & 1.243 & 4.89 \\
$\mathrm{SI}\left(\mathrm{Y}_{3}\right)$ & $81.48 \%$ & $80.56 \%$ & 1.12 \\
\hline
\end{tabular}

Notes: Bias* was calculated according to the equation: Bias $/ \%=$ (predicted valueobserved value)/predicted value $\times 100 \%$.

Abbreviations: SI, stability index; BCN-LNS, baicalin liquid nanosuspension.
Table 8 The RDI of BCN-SNS with different concentrations of sucrose after freeze-drying

\begin{tabular}{lllll}
\hline Protectants & \multicolumn{4}{l}{ Sucrose (relative to the weight of baicalin, \%) } \\
\cline { 2 - 5 } & $\mathbf{0}$ & $\mathbf{5 0}$ & $\mathbf{1 0 0}$ & $\mathbf{2 0 0}$ \\
\hline $\mathrm{RDI}$ & $1.07 \pm 0.06$ & $\mathrm{I} .05 \pm 0.06$ & $\mathrm{I} .1 \mathrm{I} \pm 0.07$ & $1.04 \pm 0.07$ \\
\hline
\end{tabular}

Abbreviations: RDI, redispersibility index; BCN-SNS, baicalin solid nanocrystals. Note: Values are mean \pm standard deviation.

Therefore, morphology evaluation of the redispersed $\mathrm{BCN}$ NS is needed. In this study, the morphology of the freshly prepared BCN-NS and redispersed BCN-NS after lyophilization was evaluated by TEM (Figure 4). TEM images of freshly prepared BCN-NS (Figure 4A) and redispersed BCN-NS (Figure 4B) were similar. This experiment revealed that the particle size of the freeze-dried BCN-SNS was distributed homogeneously as single particles. The morphology of freeze-dried BCN-SNS was evaluated by SEM. As shown in Figure 5, the BCN-SNS stabilized by TPGS postlyophilization were flocculent in shape and formed slight aggregations (Figures 5A and B). However, no significant aggregation was observed in the redispersed BCN-NS. TPGS homogeneously adsorbed on the surface of the baicalin nanocrystals and effectively prevented possible polymer-chain entanglement during lyophilization. Therefore, a slight aggregation after lyophilization did not impair the redispersibility of BCNSNS. This result also agreed well with particle-size values of redispersed NS from BCN-SNS determined by laser diffractometry (Figure 6). Therefore, the BCN-SNS after lyophilization possessed good redispersibility.

\section{Crystalline state analysis of BCN-SNS}

Crystalline state is a very important factor, which can influence the dissolution and stability behavior of a compound. High power density in the piston-gap homogenizer leads to high-energy input during the high-pressure homogenization process. ${ }^{29,30}$ In some cases, this may induce changes in the
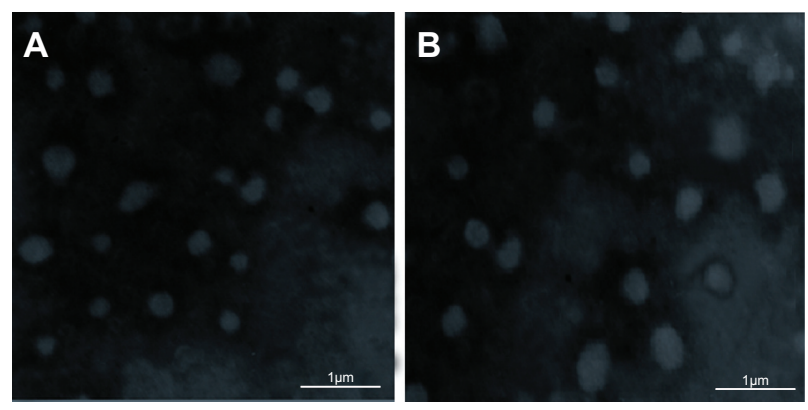

Figure 4 Transmission electron microscopy images of (A) baicalin liquid nanosuspensions freshly prepared and (B) baicalin solid nanocrystals after lyophilization treatment. 

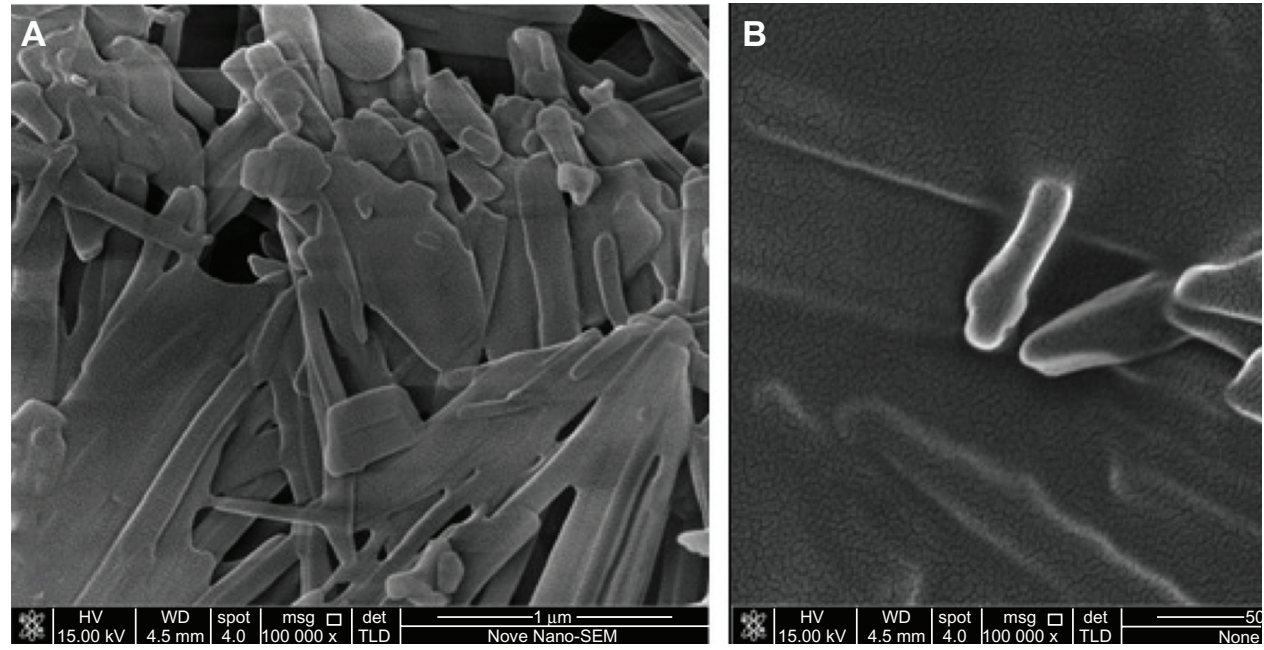

Figure 5 Scanning electron microscopy images of baicalin solid nanocrystals after lyophilization (A 5,000x; B 10,000x).

crystalline state, increasing the amorphous fraction or even creating completely amorphous particles. ${ }^{31}$ The extent of such changes depends on the chemical and on the physical hardness of the active ingredient, as well as on the applied power density. ${ }^{32}$ Therefore, before and following particle-size reduction, DSC and XRD analyses were conducted to assess if the initial crystalline state was preserved.

Figure 7 shows the XRD pattern of BCN-SNS. The powder XRD study of BCN-SNS showed no significant shift in the main peaks, compared with coarse BCN. The characteristic peak for BCN-SNS was observed at the same $2 \theta$ value as that of coarse drug. A slight decrease in intensity of peaks was observed with BCN-SNS.

The results of thermal analysis by DSC are shown in Figure 8. The diagram reveals a similarity in melting points. As shown in the DSC thermograms, the melting temperatures of $\mathrm{BCN}$ (coarse $\mathrm{BCN}$ and $\mathrm{BCN}-\mathrm{SNS}$ ) were similar. According to these results, neither the stabilizer nor the applied physical treatment affected the crystalline state of BCN.

From the DSC and XRD results (Figures 7 and 8), it was shown that $\mathrm{BCN}$ remained in a crystalline state that was physicochemically and thermodynamically more stable than $\mathrm{BCN}$ in an amorphous state.

\section{Solubility and dissolution-velocity studies of BCN-SNS}

NS could effectively enhance solubility for poorly watersoluble drugs. BCN-SNS possessed enhanced solubility $(188.6 \pm 0.33 \mu \mathrm{g} / \mathrm{mL})$ compared with the solubility $(53.51 \mu \mathrm{g} / \mathrm{mL})$ of coarse BCN. As shown in Figure 9, dissolution velocities of the freeze-dried BCN-SNS were distinctly superior compared to those of the crude powder and physical mixture. Within 60 minutes, approximately $80.87 \%$ of $\mathrm{BCN}$

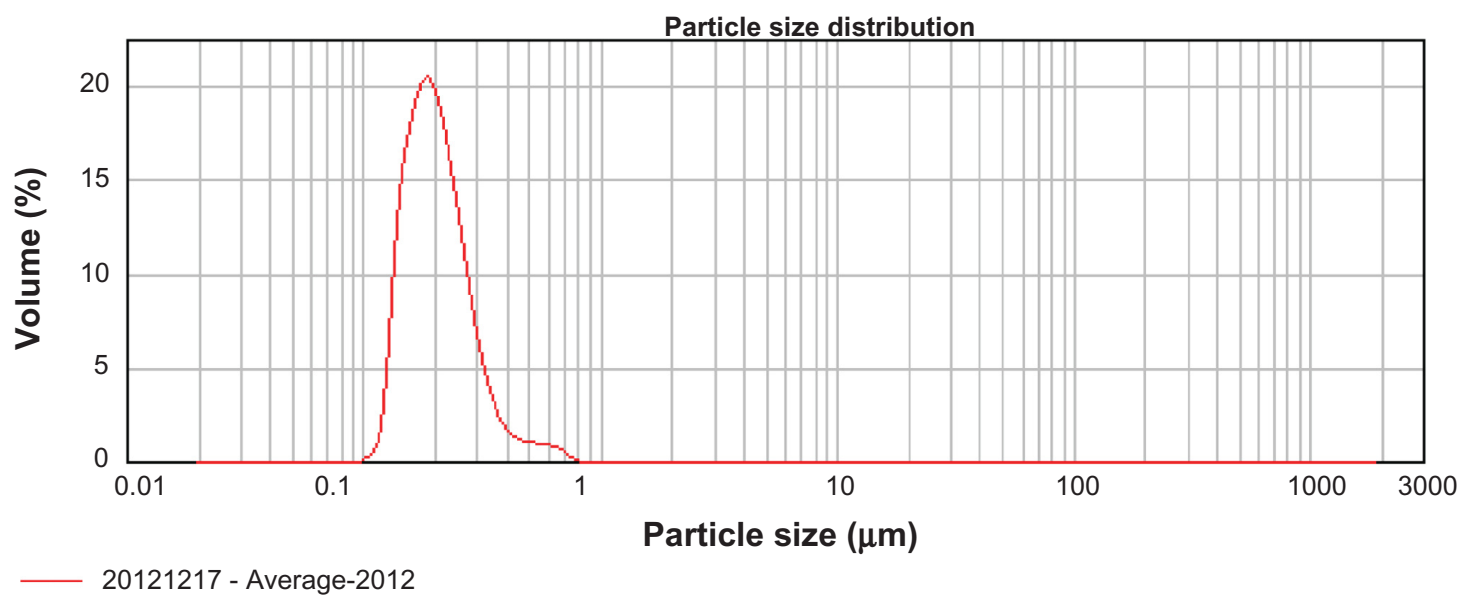

Figure 6 Particle-size distribution of redispersed baicalin solid nanocrystals (BCN-SNS). 


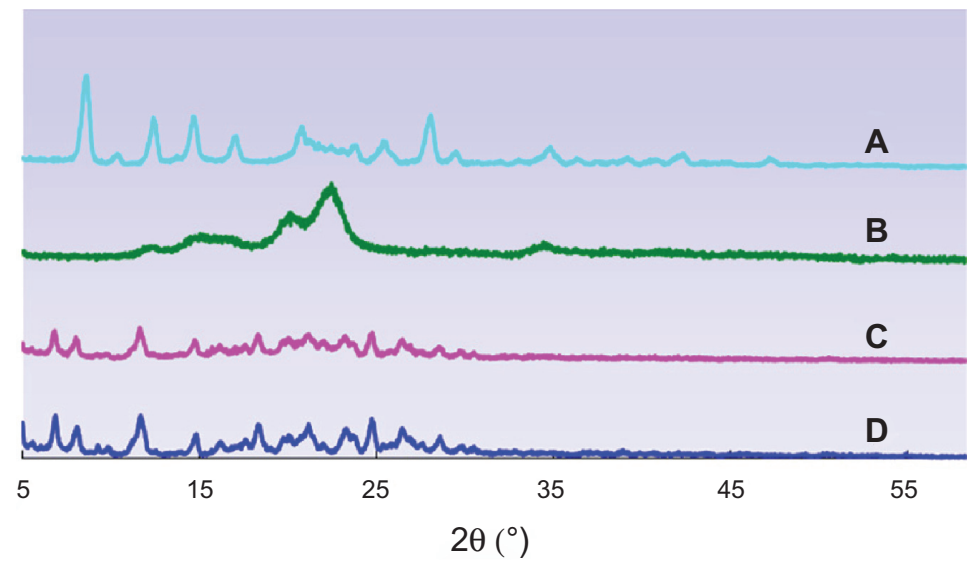

Figure 7 X-ray diffraction pattern of coarse baicalin (A), MCCS (microcrystalline cellulose and carboxymethyl cellulose sodium mixture) (B), the physical mixture (C) and baicalin solid nanocrystals (D).

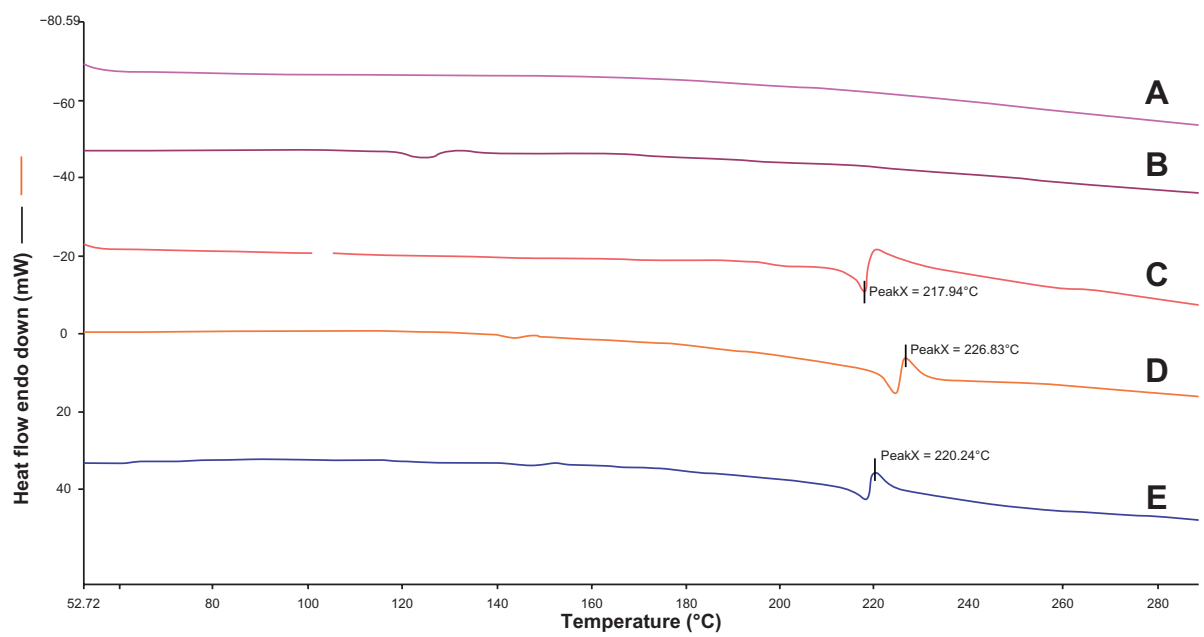

Figure 8 Differential scanning calorimetry thermograms of MCCS (A), TPGS (B), coarse baicalin (C), the physical mixture (D), and baicalin solid nanocrystals (E). Abbreviations: MCCS, microcrystalline cellulose and carboxymethyl cellulose sodium mixture; TPGS, $d$ - $\alpha$-tocopherol polyethylene glycol 1000 succinate.

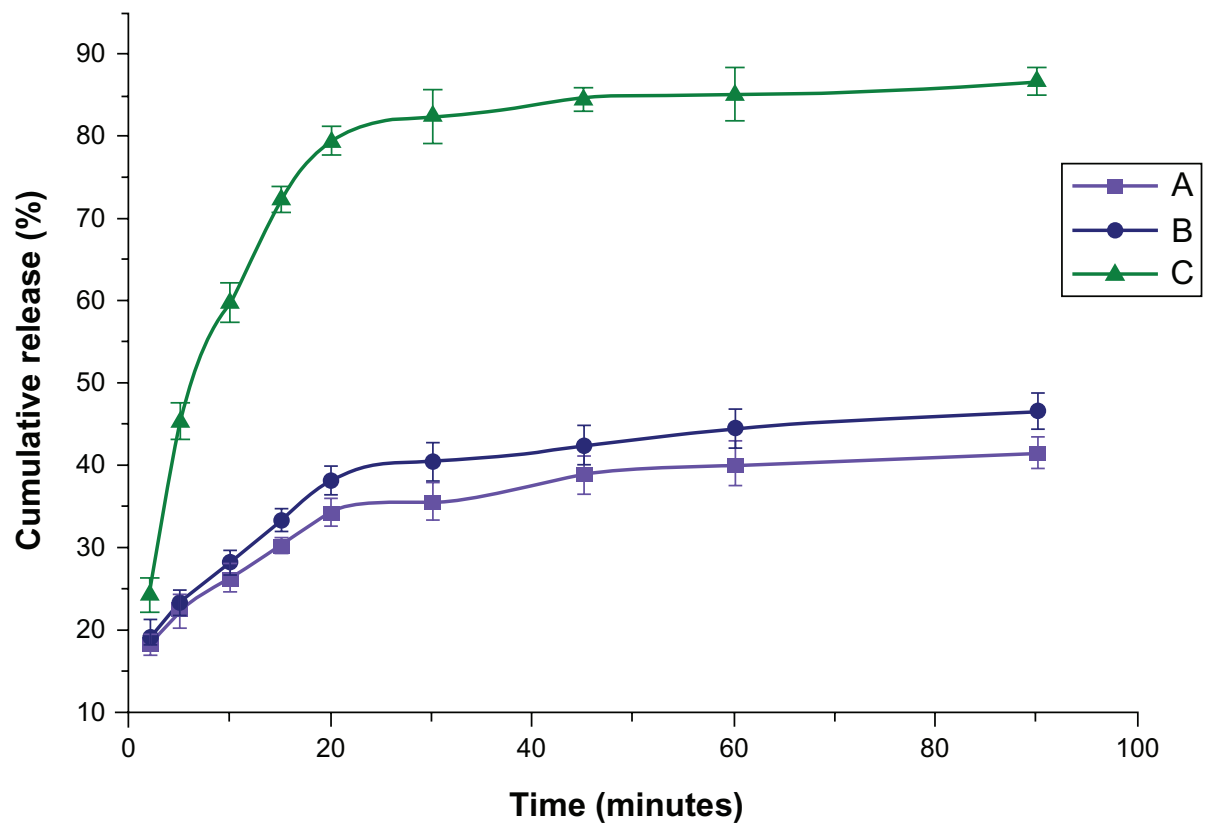

Figure 9 Dissolution profiles of baicalin coarse powder (A), physical mixture of baicalin (B), and freeze-dried baicalin solid nanocrystal powder (C). 


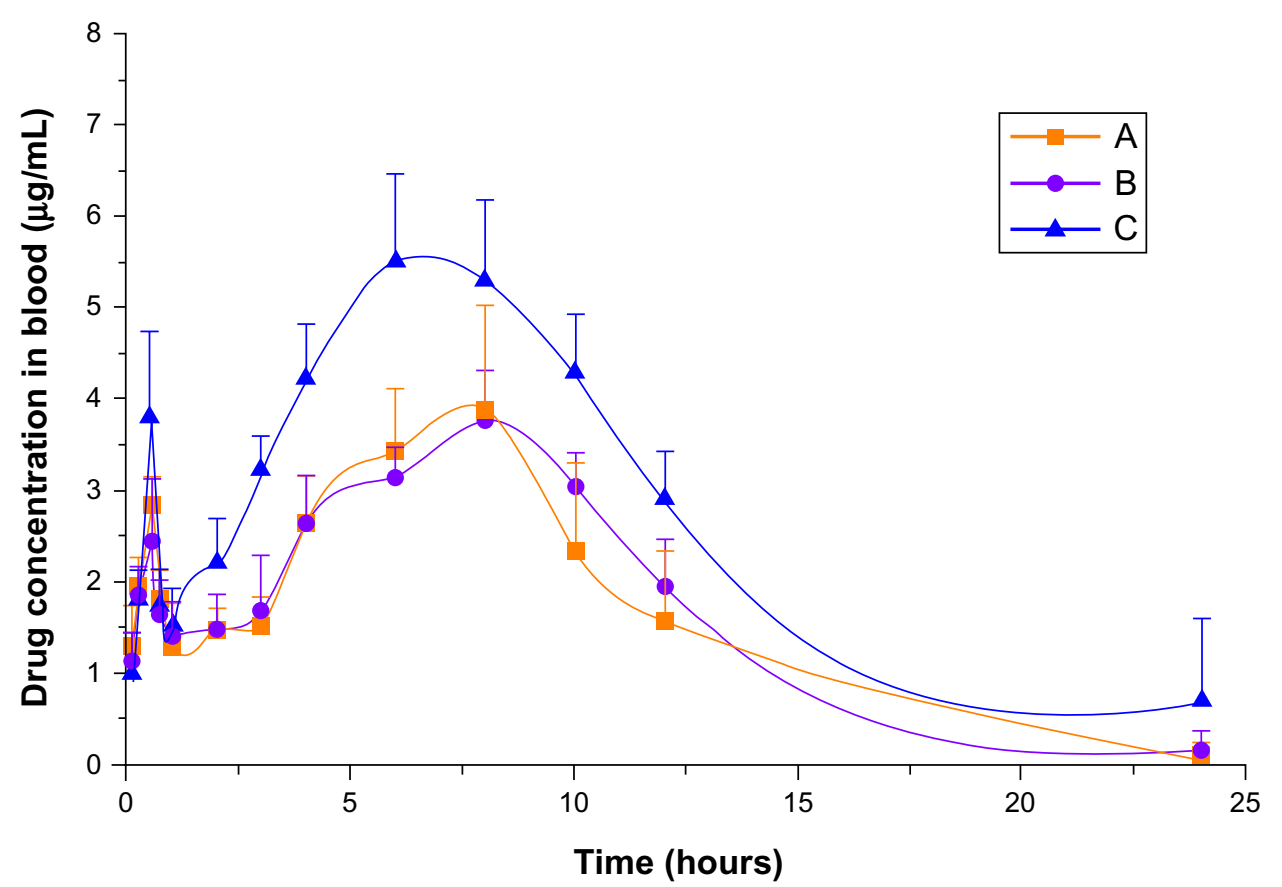

Figure 10 Mean plasma concentration-time curve of $(\mathbf{A})$ baicalin coarse suspension, $(\mathbf{B})$ baicalin physical mixture, and $(\mathbf{C})$ baicalin solid nanocrystals after oral administration $(\mathrm{n}=6)$.

was dissolved from the freeze-dried $\mathrm{BCN}-\mathrm{SNS}$. In contrast to this, only $41.15 \%$ and $43.37 \%$ was dissolved from the coarse $\mathrm{BCN}$ and physical mixture, respectively. We concluded that particle-size reduction led to enhanced dissolution velocity. This could be explained by the Noyes-Whitney equation. ${ }^{33,34}$ As when drug particles are smaller, the corresponding surface area is larger, so the nanoparticles provided a large increase in the surface area. Furthermore, according to the Prandtl equation, ${ }^{35}$ the diffusion distance, $h$, decreases for very small particles. The increase of surface area and the simultaneous decrease of diffusion distance could lead to enhancement in dissolution velocity of the drug.

\section{Pharmacokinetic study in rats of BCN-SNS}

To confirm the advantage of NS in improving the bioavailability of BCN, an in vivo pharmacokinetic study of BCNSNS was performed in rats. The plasma concentration-time curves of the BCN coarse suspension (BCN-CS) and BCNSNS are displayed in Figure 10, and the pharmacokinetic parameters are shown in Table 9. After oral administration of these three different BCN formulas, their individual mean plasma concentration-time curve profile showed a biphasic absorption phenomenon. The first absorption peak presented at about 30 minutes, and then a second absorption peak occurred at about 6 hours. This might be explained by enterohepatic circulation of $\mathrm{BCN}$, wherein $\mathrm{BCN}$ was hydrolyzed to baicalein by intestinal bacteria and then restored to its original form from the absorbed baicalein in the body, resulting in the second absorption peak. ${ }^{36,37}$

The results showed that coarse $\mathrm{BCN}$ and NS were different from each other in the corresponding parameters. Compared with the BCN-CS, the BCN-SNS had higher maximum concentration $\left(\mathrm{C}_{\max } ; 5.808 \pm 0.785 \mathrm{mg} / \mathrm{L}\right)$, higher area under the curve, 0 to time $t\left(\mathrm{AUC}_{0-\mathrm{t}}\right.$; $73.384 \pm 8.1847 \mathrm{mg} / \mathrm{L} \cdot$ hour$)$, and shorter mean residence time (10.758 \pm 1.348 hours), which indicated BCN-NS was easier to be absorbed in vivo.

BCN-NS was absorbed easily, which induced an increase in $\mathrm{AUC}_{0-\mathrm{t}}$ and $\mathrm{C}_{\max }$ and decreased mean residence time. These phenomena were probably caused by the absorption pattern of BCN. Greater exposure of the drug

Table 9 Main pharmacokinetic parameters of baicalin coarse suspension (BCN-CS) and $\mathrm{BCN}$ nanosuspension (BCN-NS) redispersed by $\mathrm{BCN}$ solid nanocrystals (BCN-SNS) after oral administration in rats $(n=6$, mean \pm standard deviation)

\begin{tabular}{llrrc}
\hline & Unit & \multicolumn{1}{c}{ BCN } & \multicolumn{1}{c}{ BCN-PM } & \multicolumn{1}{c}{ BCN-SNS } \\
\hline MRT $_{(0-\infty)}$ & hours & $8.271 \pm 0.237$ & $7.85 \pm 0.508$ & $6.758 \pm 1.348^{*}$ \\
$\mathrm{~T}_{\max }$ & hours & $7.6 \pm 0.894$ & $6.8 \pm 1.789$ & $7.2 \pm 1.095$ \\
$\mathrm{C}_{\max }$ & $\mathrm{mg} / \mathrm{L}^{-1}$ & $3.827 \pm 0.489$ & $4.212 \pm 0.805$ & $5.808 \pm 0.785^{*}$ \\
$t_{1 / 2}$ & hours & $5.26 \pm 4.36 \mathrm{I}$ & $3.223 \pm 0.864$ & $6.795 \pm 4.572$ \\
$\mathrm{AUC}_{0-24}$ & $\mathrm{mg} /$ & $43.604 \pm 4.394$ & $40.421 \pm 10.156$ & $73.384 \pm 8.184^{* *}$ \\
& $(\mathrm{~L} \cdot$ hour $)$ & & & \\
\hline
\end{tabular}

Notes: $* P<0.05$, $* * P<0.01$, versus control group.

Abbreviations: $M R T$, mean residence time; $T_{\max }$, time to maximum concentration; $C_{\max }$, maximum concentration; $t_{1 / 2}$, half-life; AUC, area under the curve. 
function group would assist uptake of the drug. Therefore, particle size has been recognized as a crucial parameter for bioadhesion and absorption from gastrointestinal tissue. ${ }^{38,39}$ The results showed that drug NS had the higher dissolution rate compared with the coarse drug. Because drug NS had a greater surface area compared with the coarse particles drug under equivalent conditions, they dissolved easily into intestinal fluid, and smaller particles showed a higher extent of uptake than larger ones via both follicleassociated epithelia and absorptive enterocytes. ${ }^{40}$

\section{Conclusion}

Process optimization of NS prepared by high-pressure homogenization was a complex process, since it involved a large number of factors that could affect the characteristics of NS. The Box-Behnken design was applied successfully to optimize formulation and process parameters for NS. From this study, it was concluded that homogenization pressure, homogenization cycles, amount of TPGS to drug, and amount of MCCS to drug played a significant role in controlling the particle size, span, and SI of NS. Homogenization pressure and homogenization cycles were considered to be significant factors that affected particle-size D50 and span of NS. The graphical optimization method helped in finding the "sweet spot" or design space to get NS with the desired physicochemical properties. This study also helped in identifying certain formulation and processing parameters, such as high polymer concentration and high homogenization pressure, which may affect the manufacturing of NS on a larger scale. The SI of the NS was found to be more dependent on polymer concentration compared to surfactant concentration.

The fabricated BCN-NS by optimization presented a sphere-like shape under TEM. SEM results demonstrated that no significant aggregation or crystal growth was observed in the redispersed $\mathrm{BCN}-\mathrm{SNS}$. By reducing $\mathrm{BCN}$ particle size into the nanometer range, its dissolution rate and oral bioavailability exhibited greater enhancement compared with coarse BCN. Through this study, it has been demonstrated that SNS might be a good choice for oral administration of poorly soluble BCN.

\section{Acknowledgments}

The authors would like to acknowledge the support from the National Natural Science Foundation of China (81102821), the Natural Science Foundation of Jiangxi Province (20122BAB215038), and the Scientific Research Foundation of Jiangxi Education Bureau (GJJ13607).

\section{Disclosure}

The authors report no conflicts of interest in this work.

\section{References}

1. Xing J, Chen X, Zhong D. Absorption and enterohepatic circulation of baicalin in rats. Life Sci. 2005;78(2):140-146.

2. Shen YC, Chiou WF, Chou YC, Chen, CF. Mechanisms in mediating the anti-inflammatory effects of baicalin and baicalein in human leukocytes. Eur J Pharmacol. 2003;465(1-2):171-181.

3. Tsai PL, Tsai TH. Pharmacokinetics of baicalin in rats and its interactions with cyclosporin A, quinidine and SKF-525 A: a microdialysis study. Planta Med. 2004;70(11):1069-1074.

4. Liversidge GG, Cundy KC. Particle size reduction for improvement of oral bioavailability of hydrophobic drugs: I. Absolute oral bioavailability of nanocrystalline danazol in beagle dogs. Int J Pharm. 1995;125(1): 91-97.

5. Liversidge EM, Liversidge GG, Cooper ER. Nanosizing: a formulation approach for poorly-water-soluble compounds. Eur J Pharm Sci. 2003;18(2):113-120.

6. Muller RH, Jacobs C, Kayser O. Nanosuspensions as particulate drug formulations in therapy: rationale for development and what we can expect for the future. Adv Drug Deliv Rev. 2001;47(1):3-19.

7. Liu ZD, Zhang XH, Wu HY, et al. Preparation and evaluation of solid lipid nanoparticles of $\mathrm{BCN}$ for ocular drug delivery system in vitro and in vivo. Drug Dev Ind Pharm. 2011;37(4):475-481.

8. Chen YJ, Jia Y, Jin RX, et al. Preparation of BCN flexible nano-liposomes. Chin J Exp Tradit Med Formula. 2011;17:1-4.

9. Wang C, Zhao XM, Hao JF, Lin J. The process on new technology and novel dosage form to improve the bioavailability of BCN. Chin Tradit Pat Med. 2012;34(3):545-549.

10. Keck CM, Müller RH. Drug nanocrystals of poorly soluble drugs produced by high pressure homogenization. Eur Pharm Biopharm. 2006;62(1):3-16.

11. Gao L, Zhang D, Chen M. Drug nanocrystals for the formulation of poorly soluble drugs and its application as a potential drug delivery system. J Nanopart Res. 2008;10(5):845-862.

12. Van Eerdenbrugh B, Vercruysse S, Martens Johan A, et al. Microcrystalline cellulose, a useful alternative for sucrose as a matrix former during freeze-drying of drug nanosuspensions - a case study with itraconazole. Eur J Pharm Biopharm. 2008;70(2):590-596.

13. Yue PF, Wang Y, Wan J, et al. [The research progress of preparation methods of solid nanocrystal delivery system.] Yao Xue Xue Bao. 2012;47(9):1120-1127. Chinese.

14. Li XS, Wang JX, Shen ZG, Zhang PY, Chen JF, Yun J. Preparation of uniform prednisolone microcrystals by a controlled microprecipitation method. Int J Pharm. 2007;342(1-2):26-32.

15. Rabinow BE. Nanosuspension in drug delivery. Nat Rev Drug Discov. 2004;3(9):785-796.

16. Van Eerdenbrugh B, Van den Mooter G, Augustijns P. Top-down production of drug nanocrystals: nanosuspension stabilization, miniaturization and transformation into solid products. Int $J$ Pharm. 2008;364(1): 64-75.

17. Patravale VB, Date AA, Kulkarni RM. Nanosuspension: a promising drug delivery strategy. J Pharm Pharmacol. 2004;56(7):827-840.

18. Singare DS, Marella S, Gowthamrajan K, Kulkarni GT, Vooturi R, Rao PS. Optimization of formulation and process variable of nanosuspension: an industrial perspective. Int J Pharm. 2010;402(1-2):213-220.

19. El-MalahY, Nazzal S, Khanfar NM. D-optimal mixture design: optimization of ternary matrix blends for controlled zero-order drug release from oral dosage forms. Drug Dev Ind Pharm. 2006;32(10):1207-1218.

20. Yue PF, Yuan HL, Li XY, Yang M, Zhu WF. Process optimization, characterization and evaluation in vivo of oxymatrine-phospholipid complex. Int J Pharm. 2010;387(1-2):139-146.

21. Yue PF, Zheng Q, Wu B, et al. Process optimization by response surface design and characterization study on geniposide pharmacosomes. Pharm Dev Technol. 2012;17(1):94-102. 
22. Yue PF, Yuan HL, Li XY, Yang M, Zhu WF, Xiao XH. Development and optimization of intravenous puerarin emulsions formation by a novel complex-phase inversion-homogenization technology. Chem Pharm Bull. 2007;55(11):1563-1568.

23. Müller RH, Gohla S, Keck CM. State of the art of nanocrystals - special features, production, nanotoxicology aspects and intracellular delivery. Eur J Pharm Biopharm. 2011;78(1):1-9.

24. Yue PF, Zheng Q, Liao MX, Zhang ZZ, Zhu WF. Process optimization, characterization, and release study in vitro of an intravenous puerarin lipid micropheres loaded with the phospholipid complex. J Dispers Sci Technol. 2010;32(1):1-7.

25. Liao ZG, Wang GF, Liang XL, Zhao GW, Jiang QY. Optimization of microwave-assisted extraction of active components from Yuanhu Zhitong prescription. Sep Purif Technol. 2008;63(2): 424-433.

26. Zheng SH, Deng YH. Application of dynamic light scattering on the evaluation of the particles size and distribution of submicroemulsion for injection. Chin J Pharm. 2005;3(3):126-131.

27. Gao L, Zhang DR, Chen MH, Zheng TT, Wang SM. Preparation and characterization of an oridonin nanosuspension for solubility and dissolution velocity enhancement. Drug Dev Ind Pharm. 2007;33(12): 1332-1339.

28. Mauludin R, Müller RH, Keck CM. Kinetic solubility and dissolution velocity of rutin nanocrystals. Eur J Pharm Biopharm. 2009;36(4-5): 502-510.

29. Möschwitzer J, Müller RH. Spray coated pellets as carrier system for mucoadhesive drug nanocrystals. Eur J Pharm Biopharm. 2006;62(3): 282-287.

30. Wang Y, Liu Z, Zhang D, et al. Development and in vitro evaluation of deacety mycoepoxydiene nanosuspension. Colloids Surf B Biointerfaces. 2011;83(2):189-197.
31. Kocbek P, Baumgartner S, Kristl J. Preparation and evaluation of nanosuspensions for enhancing the dissolution of poorly soluble drugs. Int J Pharm. 2006;312(1-2):179-186.

32. Mauludin R, Müller RH, Keck CM. Development of an oral rutin nanocrystal formulation. Int J Pharm. 2009;370(1-2):202-209.

33. Xia DN, Cui FD, Piao HZ, et al. Effect of crystal size on the in vitro dissolution and oral absorption of nitrendipine in rats. Pharm Res. 2010;27(9):1965-1976.

34. Junghanns JU, Müller RH. Nanocrystal technology, drug delivery and clinical applications. Int J Nanomedicine. 2008;3(3):295-309.

35. Pu X, Sun J, Wang Y, et al. Development of a chemically stable 10-hydroxycamptothecin nanosuspensions. Int J Pharm. 2009;379(1): 167-173.

36. Akao T, Kawabata K, Yanagisawa E, et al. Balicalin, the predominant flavone glucuronide of Scutellariae radix, is absorbed from the rat gastrointestinal tract as the aglycone and restored to its original form. J Pharm Pharmacol. 2000;52(12):1563-1568.

37. Hao J, Wang F, Wang X, et al. Development and optimization of baicalinloaded solid lipid nanoparticles prepared by coacervation method using central composite design. Eur J Pharm Sci. 2012;47(2):497-505.

38. Jung T, Kamm W, Breitenbach A, Kaiserling E, Xiao JX, Kissel T. Biodegradable nanoparticles for oral delivery of peptides: is there a role for polymers to affect mucosal uptake? Eur J Pharm Biopharm. 2000;50(1):147-160.

39. Guo JJ, Yue PF, Lv JL, et al. Development and in vivo/in vitro evaluation of novel herpetrione nanosuspension. Int J Pharm. 2013;441(1-2): 227-233.

40. Jani P, Halbert GW, Langridge J, Florence AT. Nanoparticle uptake by the rat gastrointestinal mucosa: quantitation and particle size dependency. J Pharm Pharmacol. 1990;42(12):821-826.
International Journal of Nanomedicine

\section{Publish your work in this journal}

The International Journal of Nanomedicine is an international, peerreviewed journal focusing on the application of nanotechnology in diagnostics, therapeutics, and drug delivery systems throughou the biomedical field. This journal is indexed on PubMed Central, MedLine, CAS, SciSearch $₫$, Current Contents $₫ /$ Clinical Medicine,

\section{Dovepress}

Journal Citation Reports/Science Edition, EMBase, Scopus and the Elsevier Bibliographic databases. The manuscript management system is completely online and includes a very quick and fair peer-review system, which is all easy to use. Visit http://www.dovepress.com/ testimonials.php to read real quotes from published authors. 